
subject to Institution of Engineering and Technology Copyright. The copy of record is available at IET Digital Library

\title{
Advanced DC zonal marine power system protection
}

\author{
Ke Jia, Edward Christopher, David Thomas and Mark Sumner
}

University of Nottingham, UK, NG7 2RD,

Email: ke.jia@nottingham.ac.uk

Abstract-- A new Active Impedance Estimation (AIE) based protection strategy which is suitable for utilization in a DC zonal marine power distribution system is presented. This method uses two triangular current "spikes" injections for system impedance estimation and protection when faults are detected. By comparing the estimated impedance with the pre-calibrated value, the fault location can be predicted and fault can be isolated without requiring communication between two injection units. Using cooperated double injections and line current measurement (directional fault detection), faults in the system with same impedance and different fault positions can be distinguished, located and isolated. The proposed method is validated using experimental test results derived from a $30 \mathrm{~kW}, 400 \mathrm{~V}$, twin bus DC marine power system demonstrator. The experimental tests were applied to both faults during normal operation and faults that occur during system restoration.

Index Terms-DC zonal marine system, protection, injections, impedance estimation

\section{Introduction}

The development of integrated electrical propulsion, the increase in high energy electrical loads and the widespread use of sophisticated electronics are placing a heavy demand on the quality and survivability of modern zonal marine power systems. Zonal distribution architectures can potentially be employed with a higher degree of power quality and a superior capability for fault ride-through than is presently available [1]-[4]. DC power distribution systems offer advantages over AC systems including: less conversion stages and higher efficiency; simple interface for energy storage devices and paralleling connected generators; smaller size and weight of the cables and other distribution equipment and improved flexibility [5]-[7].

The implementation of DC zonal distribution in shipboard power system will not only increase the physical burden of the protection system (such as DC breakers) [8] but also bring challenges to the existing protection scheme for short circuit faults. In order to reduce damage of short circuit faults and to provide constant voltage to loads even during system faults, "self-healing" is considered in the design of protection schemes. To realize "self-healing", the faults within each zone have to be detected, located and isolated from the healthy power system. Traditional over-current protection [9][10] cannot provide the necessary discrimination and isolate the faulted section effectively in a zonal marine system. The current differential protection [11] can remove faulted zones fast and accurately but requires communication links between terminals within the protected zone and current transducers have to be installed in each of the terminals. The communication links of the traditional current differential relay [16] reduce the survivability of the shipboard systems. Traveling wave fault location has been investigated for its speed and accuracy in distribution system [12]-[14]. However, it requires data acquisition units with very high sampling rate and the zonal architectures can increase the difficulty in applying travelling wave approaches for protection. Impedance based fault distance calculation has been introduced in [16]. However, it may be difficult to directly apply as system protection for fault location and 
isolation when faults at different positions offer the same estimated impedance.

In modern DC marine power systems, low voltage buses are supplied from AC generators and controlled rectifiers. If the load current continuously exceeds the steady current limit, or exceeds the particular transient current limit level, the output voltage and current can be quickly reduced to zero by the correct supply control strategy, so that problems associated with DC breakers can be solved. One form of this control is called "fold-back" [15] but this brings more challenges to the traditional unit protection.

This paper introduces an advance protection strategy which is based on Active Impedance Estimation (AIE). Two triangular current "spikes" are injected into the system from different zones once the system voltage drop caused by short circuit faults is detected. The estimated impedance value is then compared with the pre-calibrated system impedance value to locate the fault positions and isolate the faults. Although two injections are employed during protection they do not require any communication to make accurate operation of circuit breakers, using co-operated double injections and line current measurement (directional fault detection), faults in the system with same impedance and different fault positions can be distinguished, located and isolated. Accompany with the BIU unit, the load power supply can be maintained during the fault situation. The protection scheme works in both a normal system operating situation and a system restoration situation. It can be used as primary protection or as a backup scheme accompanied with the traditional unit protection schemes for its robust and reliability. This active impedance protection scheme is evaluated using experimental test results derived from a DC marine system demonstrator.

The paper is organized as follows. An overview of the algorithm is given in section II. The experimental set up is described in section III and the test results from normal system faults and faults during system restoration are presented in section IV. Finally the conclusions are given in section VI.

\section{Algorithm overview}

The fault protection unit which is employed in a Zonal Marine Power System (ZMPS) isolates the zone which has a short circuit fault when the system voltage dips to a value less than $90 \%$ of its rating voltage. If the system voltage is reduced for a predefined time, this indicates that a short circuit fault may be permanent (or if use AIE protection as a backup strategy, that the primary protection may have failed). A typical 5 zone (separated by circuit breakers) ZMPS is shown in Fig. 1. Loads can be supplied from the port bus or the starboard bus (port bus is the primary power supply bus and the starboard bus is the alternative one). Two current injections (from different zones) are carried out and the faults can be located by calculating the measured system impedance from the injection point. Since most of the short circuit faults within the ZMPS have a very low fault impedance, the measured system impedance is dominated by the fault loop impedance (the bus bar impedance from the injection

point to the fault point) characteristics. By comparing the estimated impedance with pre-calibration value, the fault distance can be estimated and the tripping signal to open the appropriate Circuit Breakers (CB) for fault isolation can be initiated. 


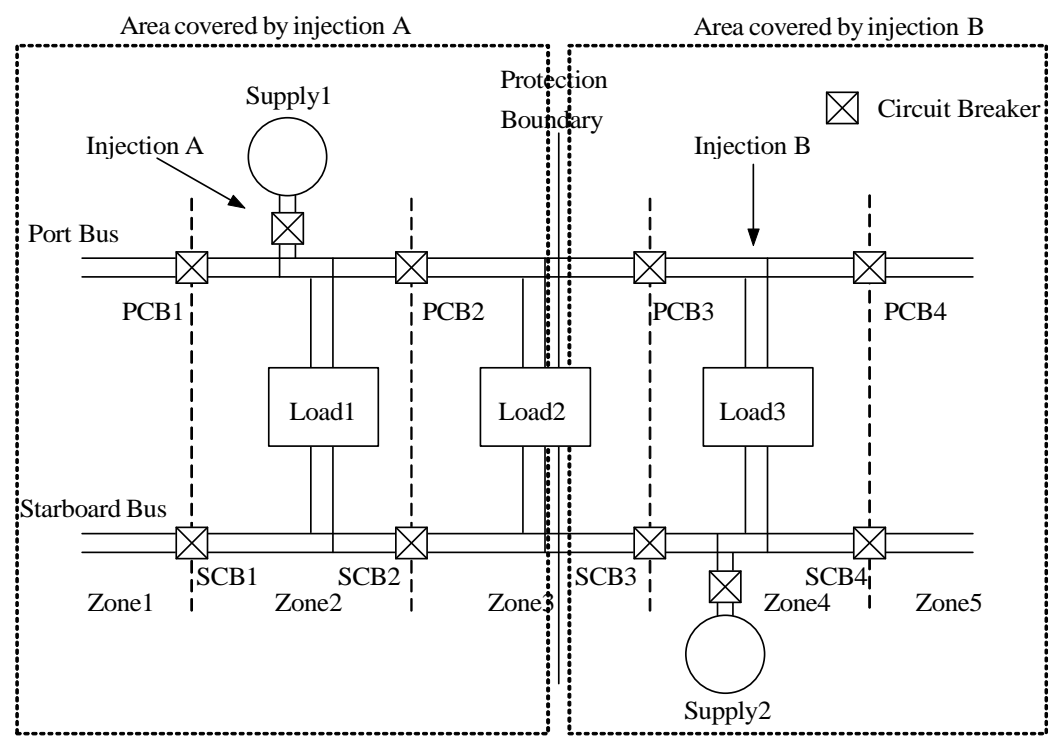

Fig.1 Simplified DC zonal distribution system

At each injection point the injection voltage, injection current and the bus current are measured. By using the bus current measurement, faults direction (by the left or right side of the injection) can be predicted. Once the fault is detected, the foldback\| unit brings down the fault current by controlling the DC output current of the supply rectifier. Measuring the bus injection current direction can provide the correct information of fault direction. The fault location is then based on distance estimation and direction estimation. If the estimated distance indicates the fault is near the other injection unit then the tripping decision is left to the other unit. Only after a pre-defined delay without any noticeable system recovery will the injection unit furthest from the fault instigate CB tripping and fault isolation. For example, consider a fault on the port bus of Fig.1, Injection unit A covers the left side area of the boundary (solid black line) and injection unit B covers the right side. If a fault occurs near injection unit B in Zone 4 (by the right side of the boundary) injection unit A will estimate the fault distance and direction to indicate the fault is near injection B and initially leave the tripping to Injection unit B. The protection scheme within each injection unit can be summarized as 1) detecting the fault (using bus voltage sagging); 2) detecting the fault direction (using bus injection current measurement); 3) estimate the fault distance; 4) if fault is out of the protection zone delay the circuit breaker tripping signal (leave the fault to the closer injection unit) 5) if after the delay bus voltage is not recovering (the other injection unit fails) trips the breaker and isolate the fault.

Injecting positions are selected to primarily provide protection to the system generators and important loads. For stand-alone injections (as discussed in this paper), the injection units are installed close to system generators to guarantee the voltage supply safety. For injections that combined with converters, the injection points can be at the both the supply and the load converters. Using two co-operated injections (each injection covers the close zones as described in Fig.1) and line injected current directional measurement. The faults with same impedance at different positions can be distinguished. A detail example is discussed in Section 4. 
The injection is performed by a controlled grid connected half bridge circuit with a DC link capacitor through a coupling inductor as shown in Fig 2.a. The ideal injection voltage $\left(\mathrm{V}_{\text {Inj }}\right)$ and the created injection current ( $\left.\mathrm{I}_{\text {Inj }}\right)$ throught the coupling inductor are shown in Fig. 2b. A step voltage waveform is created by controlling the operation of the two IGBT switches and is applied to the coupling inductor resulting in a triangular current. The DC link capacitors are four 400V (DC), $2.2 \mathrm{mF}$ in series connected to stand the applied 1000V DC link voltage. This current is then injected into the system through the Point of Common Coupling (PCC).

a)

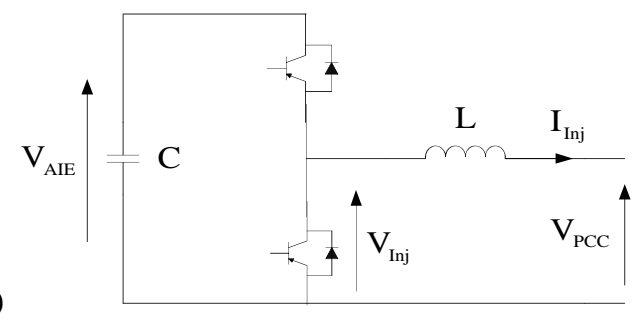

b)

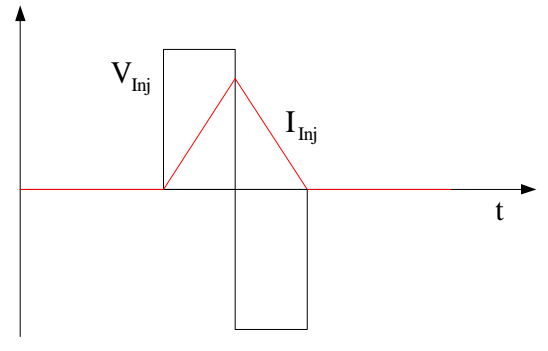

Fig. 2 Injection unit and its Injection voltage and current

The voltage and current are recorded and processed by a DSP with the following procedure: Blackman [16] windowing; Zero padding; Fast Fourier Transform (FFT), Impedance calculation Z=FFT(V)/FFT(I) and Curve fitting as shown in Fig. 3

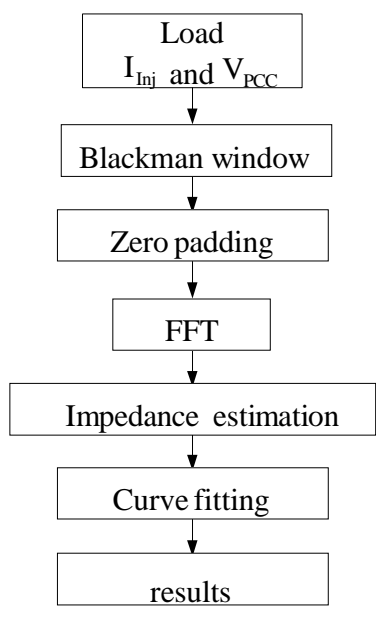

Fig. 3 Procedure of active impedance estimation

The algorithm of fault distance estimation can be presented in 5 steps: 1) The measured voltage and current are Blackman windowed as equation (1) to remove the edge influence of the recorded data. This will create a smooth curve after the data is processed with FFT. 


$$
w(n)=0.42-0.5 \cos \left(\frac{2 \pi n}{N-1}\right)+0.08 \cos \left(\frac{4 \pi n}{N-1}\right)
$$

As presented in (1), $\mathrm{N}$ is the length of the Blackman window. Edges of recorded data section (rectangular window) will cause waveform distortion after directly transformed to frequency domain. The Blackman window which smoothes the recorded data edges to zero will eliminate this affect and also provide a good condition for data zero padding; 2) Zero padding doesn't add any information to the original data but increase the length of the data with zeros. This provides a better frequency resolution and will improve the fault distance estimation accuracy accompany with the curve fitting process; 3) The padded voltage and current data is transformed into frequency domain by Fourier Transform:

$$
F(x(n))=\sum_{n=0}^{L-1} x(n) e^{-(2 \pi i n k \Delta t / L)}
$$

where $\mathrm{n}$ is the sample index, $\mathrm{k}$ is the frequency index and $\Delta t$ is the sample period. The can be make more efficient by using the FFT where only the frequency steps of $L / \Delta t$ are taken ( $\mathrm{k}$ is an integer) and it is assumed that the signal is infinitely repeating or finite extent [18]-[19]; 4) The system impedance is calculated in the frequency domain using (3) and the reactance part (dominate part) is used for fault distance calculation.

$$
X(f)=\frac{\operatorname{imag}(F(v(t)))}{\operatorname{imag}(F(i(t)))}
$$

5) Due to the system steady state distortion and background measurement noises, the estimated reactance waveform in the frequency domain has some oscillation. A least square curve fitting which provides a fitted straight line to given points in frequency domain is applied the impedance results for better accuracy [17]. In order to unilize a linear least squares curve fitting to a $n$ points sampled data, the vertical offset $R^{2}$ of $n$ points is defined as:

$$
R^{2}=\sum_{i=1}^{n}\left[y_{i}-\left(a+b x_{i}\right)\right]^{2}
$$

where $x$ is frequency information and $y$ is the correspond reactance value. Assuming the fitted line is $y=a+b x$. In order to minimize the deviations:

$$
\begin{gathered}
\frac{\partial\left(R^{2}\right)}{\partial a}=-2 \sum_{i=1}^{n}\left[y_{i}-\left(a+b x_{\imath}\right)\right]=0 \\
\frac{\partial_{\text {«- }}}{\partial b}=-2 \sum_{i=1}^{n}\left[y_{i}-\left(a+b x_{\imath}\right)\right] x_{i}=0
\end{gathered}
$$

Solving the (5) and (6), $a$ and $b$ can be calculated using (7)

$$
\left[\begin{array}{l}
a \\
v
\end{array}\right]=\left[\begin{array}{cc}
n & \sum_{i=1}^{n} x_{i} \\
\sum_{i=1}^{n} x_{i} & \sum_{i=1}^{n} x_{i}^{2}
\end{array}\right]^{-1}\left[\begin{array}{c}
\sum_{i=1}^{n} y_{i} \\
\sum_{i=1}^{n} x_{i} y_{i}
\end{array}\right]
$$


Then the estimated line reactance results (from the fitted line) are compared with the pre-calibration value to estimate the fault distance. The fault distance information is then used during the protection as described at the beginning of this section.

\section{Experimental setup}

A $30 \mathrm{~kW}, 400 \mathrm{~V}$, twin bus DC zonal distribution demonstrator has been constructed to validate the proposed protection scheme. The demonstrator has five zones and two independent DC supplies which contain synchronise generator (driven by induction motor), controlled thyristor rectifier and DC line filter. As shown in Fig. 4, normally Supply 1 is connected to the Port bus and Supply2 is connected to the starboard bus.

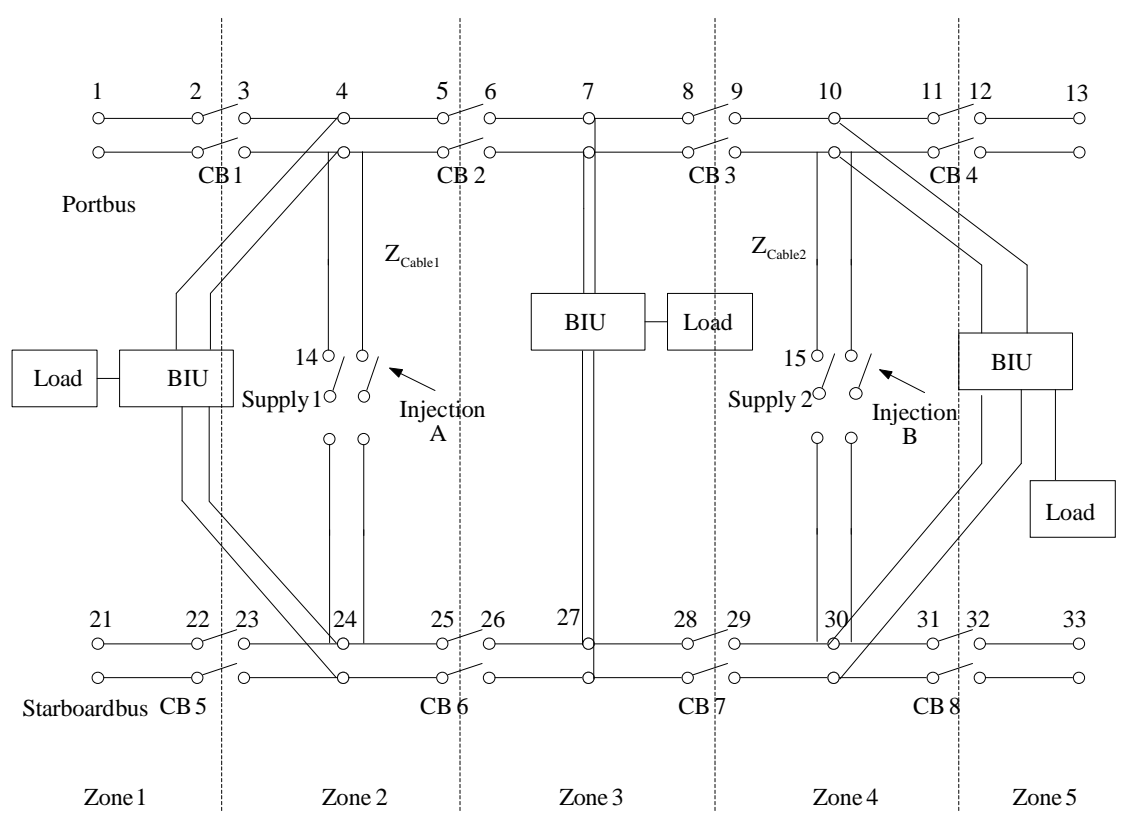

Fig. 4 30kW, 400V, twin bus DC demonstrator

Loads are connected in Zones 2, 3 and 4 through the Bus Interface Unit (BIU) to either port bus or starboard bus. The BIU involves controlled power electronics and decides whether the load is connected to port bus, starboard bus or a energy storage unit during a short circuit fault situation to maintain a continuous power supply. In this demonstrator, initially all the loads are connected to the Port bus and when faults occur on the Port bus loads are switch to an energy storage unit during the fault and then to the other bus using the BIU. Details of the demonstrator are shown in Fig. 5. 


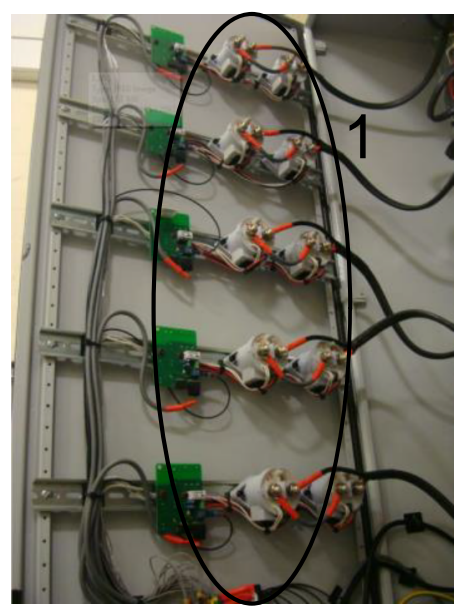

(a)

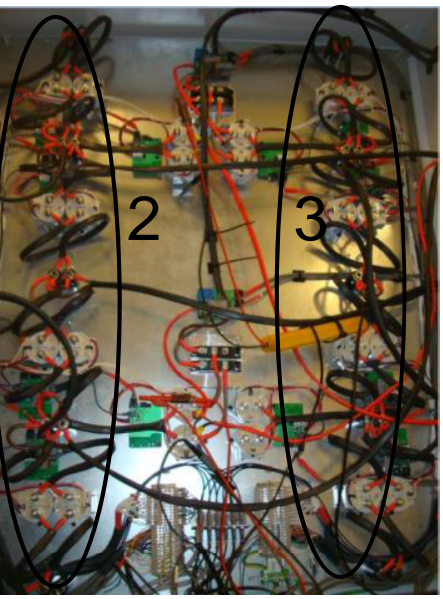

(b)

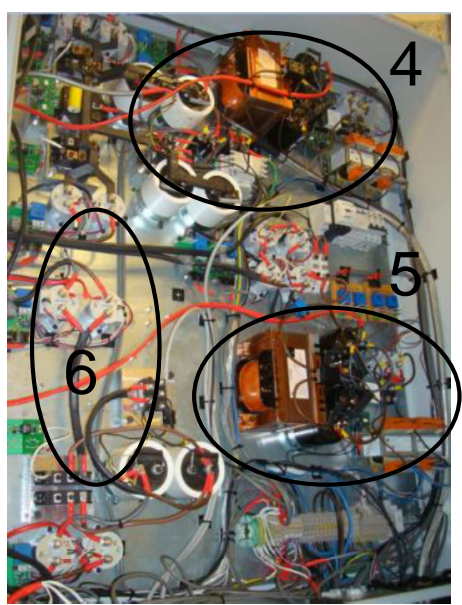

(c)

Fig. 5 (a) Fault units. (b) DC network demonstrator: 2) Port bus 3) Starboard bus. (c) BIU and Injection: 4) Injection A, 5)

Injection B, 6) BIU interface.

All the Circuit Breakers (CB), in Fig. 5 (b), are managed by a DSP with protection logic embedded. A 10mm single core cable is used to simulate the bus bar in the marine power system. Each section of the cable represents a $10 \mathrm{~m}$ bus bar which has inductance of approximately $0.4 \mu \mathrm{H}$. The BIU, which contains boost converter (provides the rated bus voltage to loads during the system fault) and thyristor switches, is operated by the DSP signal once the bus voltage sag is detected. The DC voltage (1000V) across the DC link capacitors of the injection unit is supplied from a $230 \mathrm{~V}$ AC main through two step-up transformers and a diode rectifier. The injection coupling inductor is $6.8 \mathrm{mH}$ to maintain the peak of the injection current to be within $30 \mathrm{~A}$. The five fault units contain controlled contactors and short piece of cable can be used to imposing short circuit faults on the different positions of the two buses.

\section{System impedance calibration and BIU test}

For the proposed protection scheme, faults can be located by comparing the online estimated impedance value with the calibration value. The fault location accuracy is based on accurate system impedance calibration on a de-energized system. Because the Port bus and the Starboard bus are symmetrical and the Port bus is the primary power distribution bus, only Bus A is calibrated. As shown in Fig. 4, short circuit faults are initiated at positions 1 to 15 and bus impedance value seen from the two injection points are calculated. For each injection point, three current "spikes" are injected and the average impedance value are derived. The typical injected current and voltage response measured at $\mathrm{V}_{\mathrm{pcc}}$ for a fault at location '8' in Fig.4 are presented in Fig.

6. 

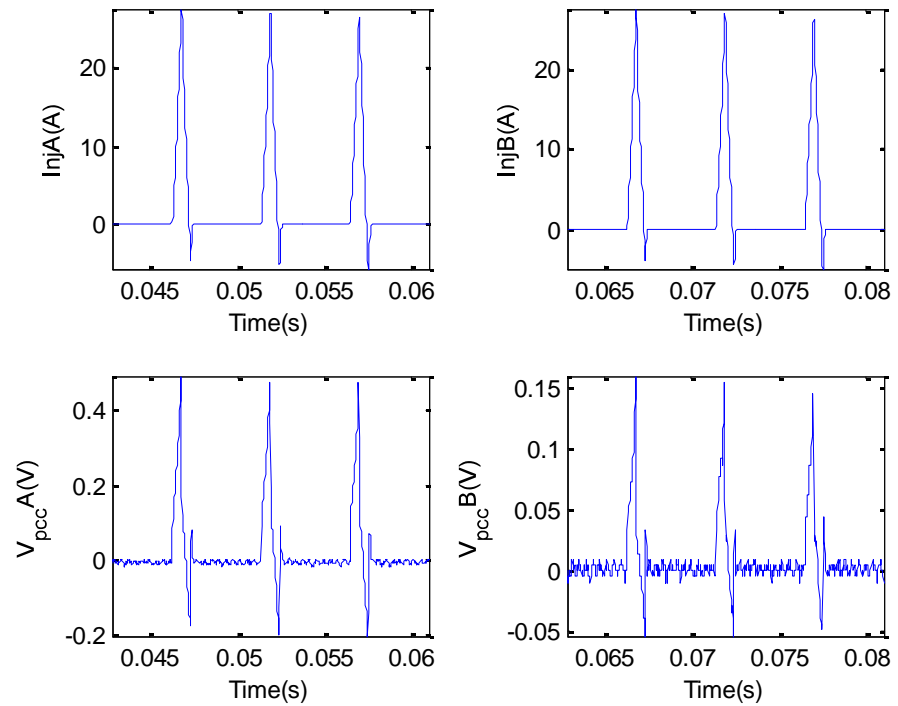

Fig. 6 Injection current and voltage from two injection points

The injected current is kept within 30A amplitude and $1 \mathrm{~ms}$ duration in time to maintain minimum disturbance to the system and also provide good signal to noise ratio (SNR) for impedance calculation. As the distance between fault (at ' 8 ' in Fig. 4) and the two injection points are different, the voltage response varies as shown in Fig. 6 . The recorded voltage and current are then processed using the procedure shown in Fig. 3. The typical unified ABS value of the injection current and its voltage response are presented in frequency domain in Fig.7. The estimated reactance results in the frequency domain are shown in Fig. 8.
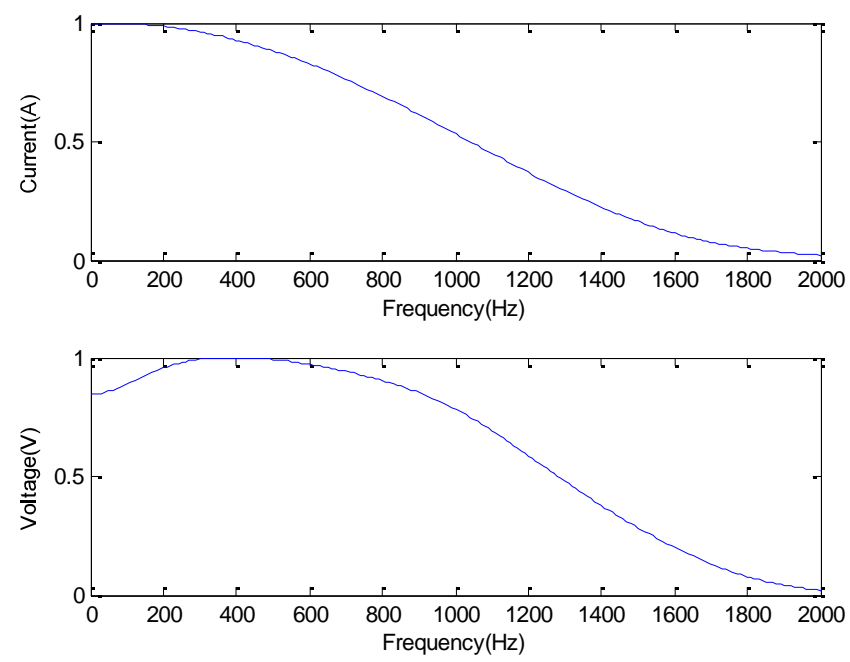

Fig. 7 Measured injection current and its voltage response in frequency domain 

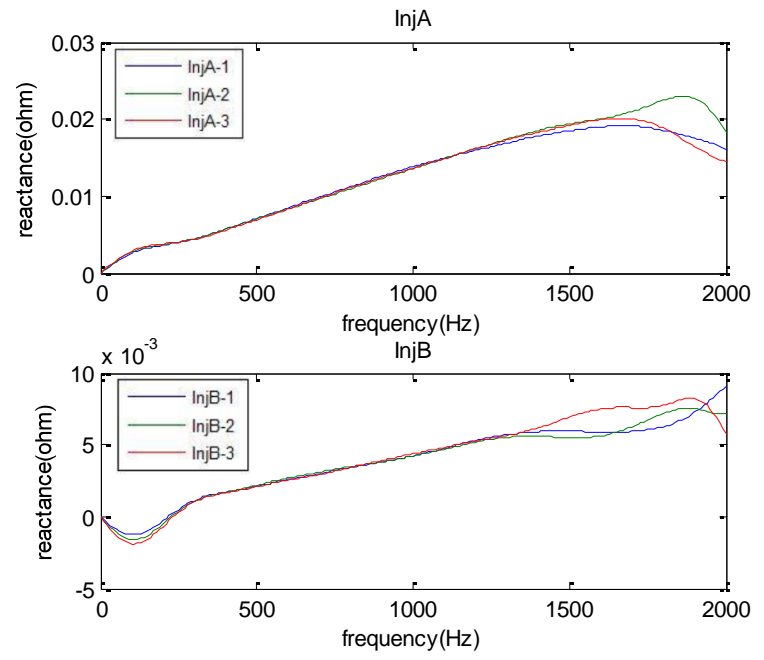

Fig. 8 Reactance values estimated from Injection A and Injection B in frequency domain

As shown in Fig. 8, the impedance results are demonstrated in a frequency range up to 2000Hz. According to the frequency characteristic of the injection current and its voltage response in Fig.7, the frequency range (2000kHz in this paper) is chosen to get good SNR, to be within the bandwidth of standard instrument, to restrict sample frequencies to those used in standard data acquisition equipment and also to be able to ignore system parasitic capacitance effects. The injected 1ms triangular current signal attenuates significantly in the high frequency range (above $1500 \mathrm{~Hz}$ ) as demonstrated in Fig.7. The low frequency (below 300Hz) variation is mainly caused by the Blackman windowing. The estimated reactance values have good consistency and SNR in the frequency range between $400 \mathrm{~Hz}$ and $1300 \mathrm{~Hz}$. This frequency range is select due to the frequency characteristic of the injection waveform and it can be applied to the different systems. Through testing, it has been found that this frequency range provides best accuracy in the protection scheme. The estimated line inductance (in $\mu \mathrm{H}$ ) for all the faults positions from the two injection points are shown in Fig. 9.

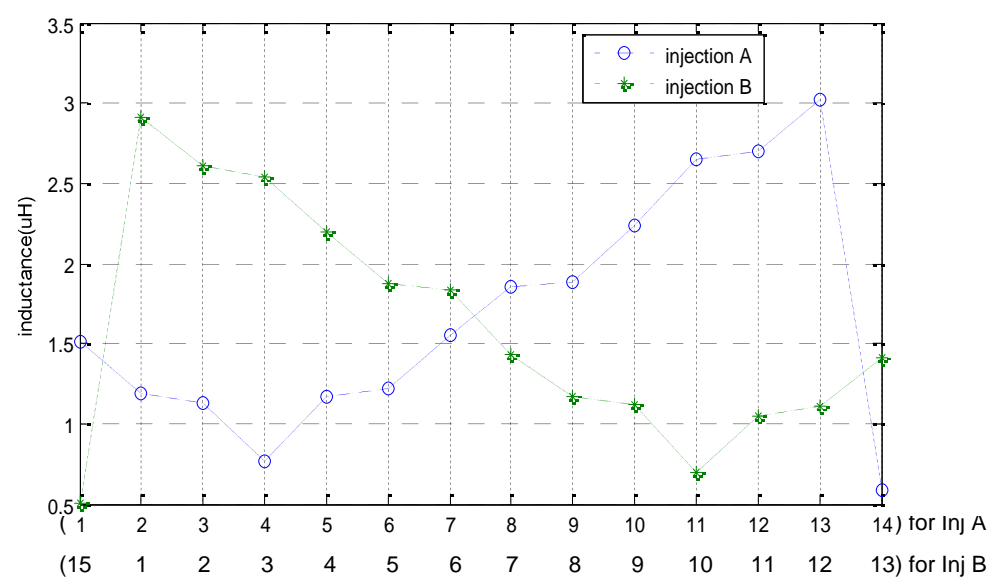

Fig. 9 Inductance value as seen from injections points A and B for all the fault positions on Bus A

Fig.9 is created by applying short circuit faults to all the positions on the port bus and calculating the inductance value between the injection and the fault points. For the demonstrator structure shown in Fig. 4, the inductance values seen from the two 
injection point for all the possible fault locations are nearly symmetrical around the injection points. The cable connected Injection unit A to the bus is slight longer than the cable used to injection unit B and this leads to the injection unit A sees a larger inductance for faults at symmetrical positions to the injection B. For example, fault at 14 for injection A offers larger inductance than 15 for injection B. The small inductance difference between 2-3, 5-6, 8-9 and 11-12 seen by injection A (and injection B) is induced by pair of contactors (and their connection points) which are used as circuit breaker in the demonstrator. The slight mismatch is also due to the difference in connection impedance. The calculated inductance value will be used as a reference for locating the fault position in the protection scheme. Although the different fault locations (on both sides of the injection point and have same distance to the injection) may offers similar inductance value (3,5 for injection A and 9 , 11 for injection B) using the line injecting current measurement as mentioned in section2 (protection algorithm) the fault direction and fault distance can be located. The protection scheme can successfully isolate the faulted section of the system after fault detection and location while the BIU units maintain the load voltage supply during the short circuit fault situation where the bus voltage drops. This is demonstrated, in an energized system, by imposing short circuit fault in Zone 2 of the system and the recorded supply voltage (from supply1), port bus voltage and BIU voltage (load voltage) are shown in Fig. 10.

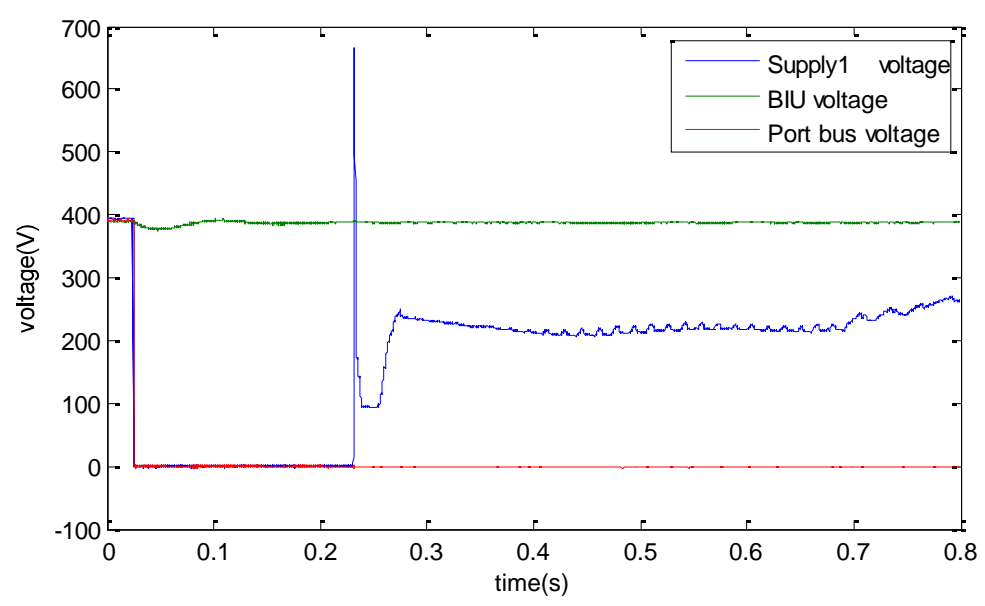

Fig.10. Measured system voltage during a short circuit fault

The system (as shown in Fig. 4) is supplied with 400V DC through supply 1 for normal operation and all three loads are connected to the port bus. Fig.10 shows only the typical voltage response of BIU1 for the fault situation as all the three BIUs are identical. The fault occurs at about 0.02 s and causes the supply voltage (red) and the bus voltage (blue) to drop significantly. Once the BIU detects a voltage drop, it will start operating and control the converter to meet the output voltage (load interface voltage) with the preset rated value $(400 \mathrm{~V})$. As is shown by the green curve in Fig. 10, although with slight oscillation at the initiation of the fault, the BIU can keep its output voltage value close to $400 \mathrm{~V}$ during fault. After the fault is detected and isolated (at about 0.23s), breakers CB1, CB2 and the supply1 breaker open, the supply1 voltage is starting to recover. However, due to fact that the fault is located in the generator zone, the supply1 circuit breaker will not be closed unit the fault is removed and the cable is repaired. So the port bus voltage remains zero. "Self-healing" in this system is realised according to the fact that the fault in the system is detected, located and isolated and at the same time load voltage is not interrupted during the fault. 
The fault protection scheme is activated at the same time as bus voltage drop is detected. It takes about 200ms to clear the fault (this includes the DSP processing time, circuit breaker operating time and the necessary delay for injections) and at about $0.22 \mathrm{~s}$ zone 2 is isolated and the supply is disconnected due to the fact that the fault and the supply are in the same zone. The supply voltage (blue line) begins to return to its rated value because the supply CB is tripped the bus voltage will be zero and maintain for a short period of time (to make sure the fault is isolated and start the other supply).

\section{Experimental test results on an energised system}

\section{A. Results for faults in normal system}

The DC demonstrator is symmetrical between the port bus and starboard bus. The experimental tests are carried out on the port bus for initial work. The rig can be simplified as shown in Fig. 11.

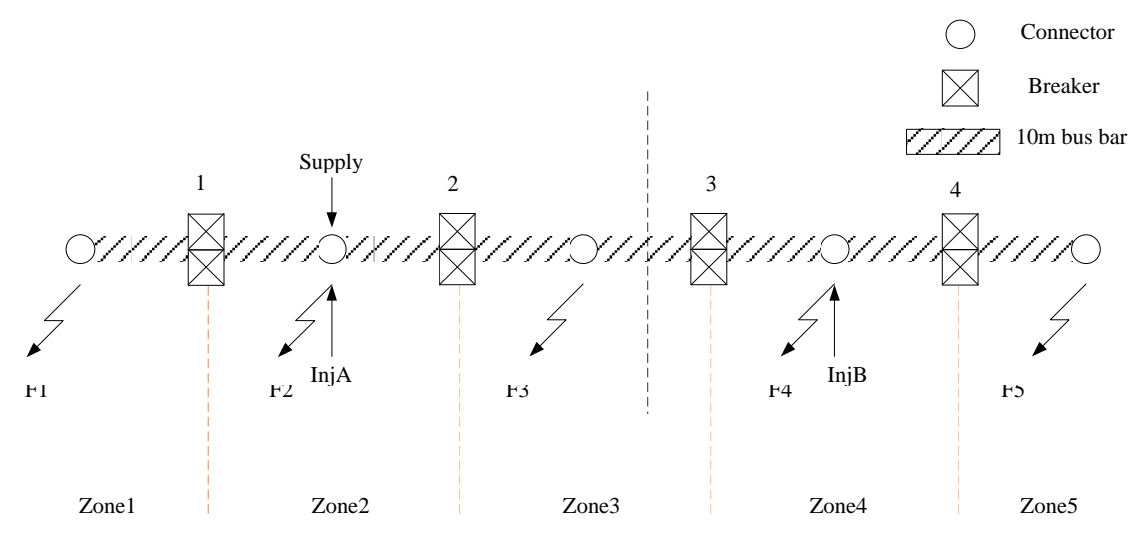

Fig. 11 Simplified DC demonstrator

Short circuit faults are placed in each zone to test the proposed protection scheme. The cable inductance between each section (approximately $0.4 \mu \mathrm{H}$ ) represents a 10m length bus bar in the real DC marine power system. For fault F1, the estimated reactance and the inductance values are as shown in Fig. 12.
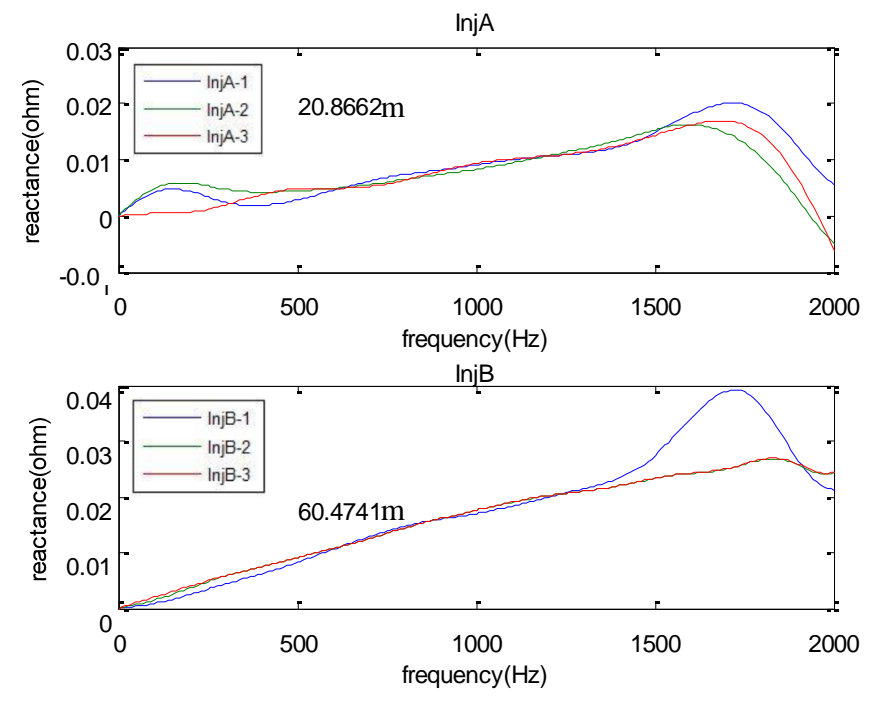

Fig. 12 The estimated reactance and resultant inductance value for fault F1 in $400 \mathrm{~V}$ energised system 
Compared to the calibration results derived from the de-energized system in Fig.8, the energized test is influenced by system noise. There is larger oscillation within the estimated curve and there is a consistent error. However, this error (within $3 \%$ in frequency range $400 \mathrm{~Hz}-1300 \mathrm{~Hz}$ ) is acceptable for the required accuracy. The numbers above each of the reactance curve are the estimated fault distance (in metre) seen from the injection points. These fault distance results (presented in the tables) are derived by dividing the average value of the three injection results by the pre-calibrated inductance value in per-metre length of cable as shown in (8)

$$
\mathrm{D}=\frac{\sum_{\mathrm{n}=1}^{\mathrm{N}} \mathrm{H}_{\mathrm{n}}}{\mathrm{N} \times \mathrm{H}_{\text {per-unit }}}
$$

where $\mathrm{D}$ is the estimated fault distance. $\mathrm{N}$ is the number of injections. $\mathrm{H}$ is the estimated inductance value and $\mathrm{H}_{\text {per-unit }}$ is the calibrated inductance value per-metre.

The injections take place about $10 \mathrm{~ms}$ after the continuous voltage drop caused by fault is detected. During this time, the system bus voltage is very small (as shown in Fig. 10) and so is the noise and this is true for systems with even higher voltage supply. The system noise and distortion caused by non linear load which may appear in the healthy operation are not a serious problem during short circuit faults for impedance estimation. The "fold-back" algorithm within the supply rectifier controls the DC output current to a very small value (about $1.5 \mathrm{~A}$ in here) once the fault is detected. There are two current transducers located by each side of the injection unit on the bus. If a current larger than 10A (30A peak for the injection current) is detected and the current lasts for more than $0.3 \mathrm{~ms}$ by one of the current transducer, the fault is at that transducer side. Using this criterion the fault F1 and F3 for injection A (or F3 and F5 for injection B) can be distinguished. Compared with the actual fault distance which is $20 \mathrm{~m}$ left of injection point $\mathrm{A}$ and $60 \mathrm{~m}$ left of injection point $\mathrm{B}$, the estimated results are accurate enough to locate the faults within each zone. Table 1 shows the fault direction (by measuring the fault current) and fault distance estimation results and the breaker operation for all the five faults.

\begin{tabular}{|c|c|c|c|c|c|}
\hline & F1 & F2 & F3 & F4 & F5 \\
\hline Distance by InjA & 20.86 & 0.61 & 21.55 & 40.93 & 63.51 \\
\hline Direction by InjA & left & right & right & right & right \\
\hline Distance by InjB & 60.47 & 39.48 & 19.86 & 0.9 & 19.89 \\
\hline Direction by InjB & left & left & left & left & right \\
\hline Relay based on & InjA & InjA & InjA & InjB & InjB \\
\hline Fault locaiton & zone1 & Zone2 & Zone3 & zone4 & zone5 \\
\hline Breaker open & 1 & 1,2, & 2,3 & 3 & 4 \\
\hline
\end{tabular}

Table 1 Fault location results and circuit breaker behavior

As presented in Table 1, for all the faults, the fault distance is estimated accurately (error within 3\%). The right circuit breaker tripping decision made by the protection scheme and the faults can be correctly isolated. As expected, when the fault 
distance seen from Injection A is less than $26 \mathrm{~m}$, the breaker operation decision is made based on the fault location results from Injection A and for fault further than $26 \mathrm{~m}$ (seen from Injection A), breaker operation is controlled by Injection B.

\section{B. Results for faults during system restoration}

Once a fault is detected and isolated, the load will be switched to the BIU for a short period and then switched to the health bus. The faulted section (on faulted bus) will be disconnected and replaced with cable. The system working with one section of the bus bar being replaced with cables which have about 5 times larger impedance after system restoration. The proposed protection scheme will need to work for the new system configuration as well.

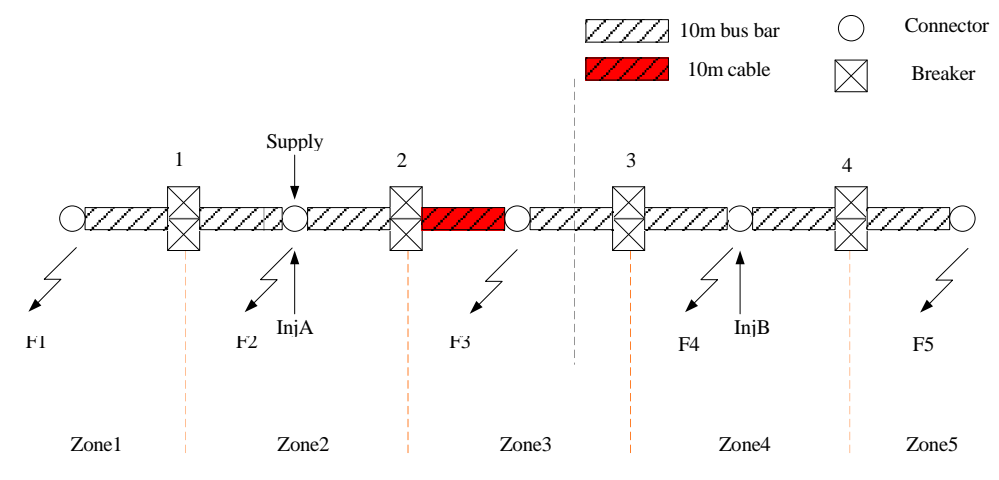

Fig. 13 DC demonstrator with one section busbar replaced by cable

An example of a system configuration which has one section of bus bar replaced with cable is shown in Fig. 13, the 10m busbar between circuit breaker 2 and fault3 (F3) is replaced with cable which has approximately 5 times (4.6 times in the calibration) larger impedance. If this bus is used and another fault occurs on the bus, the protection scheme will still trigger the correct breaker to isolate the faulted section according to the pre-set algorithm as described in Section II.

\begin{tabular}{|c|c|c|c|c|c|}
\hline & F1 & F2 & F3 & F4 & F5 \\
\hline Distance by InjA & 20.57 & 0.75 & 55.91 & 76.05 & 97.12 \\
\hline Direction by InjA & left & right & right & right & right \\
\hline Distance by InjB & 96.27 & 75.85 & 20.21 & 0.56 & 19.54 \\
\hline Direction by InjB & left & left & left & left & right \\
\hline Relay based on & InjA & InjA & InjA & InjB & InjB \\
\hline Fault locaiton & zone1 & Zone2 & Zone3 & zone4 & zone5 \\
\hline Breaker open & 1 & 1,2 & 2,3 & 3 & 4 \\
\hline
\end{tabular}

Table 2 Fault location results and circuit breaker behavior when bus bar between CB2 and F3 is replaced by cable

As shown in Table 2, for faults on the left side of the circuit breaker 2 (F1 and F2 in Fig. 13), the cable section is not in the loop that the current of injection A flows through. The estimated fault distance from Injection A is correct. Because the fault distance seen from Injection A is less than $26 \mathrm{~m}$, the circuit breaker operation is decided by using the fault location results derived from injection A. For faults on the right side of the cable section (F3, F4 and F5 in Fig. 13), the fault location results produced by Injection $\mathrm{A}$ is not correct due to the fact that the cable section which has much larger impedance value than a bus bar is within the loop of Injection A. However, the Injection A will think these faults are out of its protection area (within 26m) and the relay final 
decision will be made using the correct fault location results estimated by Injection B. By this way, faults on all positions of the bus can be located correctly and isolated.

If fault occurs on the cable section, than both Injection A and Injection B will estimated the fault is out of their protection zone. If after the fault detection the bus voltage is still not restored, as the fault is on the cable section and breaker at both ends of the cable section will be tripped.

Tables listed below shows that although different sections of bus bar are replaced by cables the protection scheme is able to trip the correct circuit breakers when fault occurs.

\begin{tabular}{|c|c|c|c|c|c|}
\hline & F1 & F2 & F3 & F4 & F5 \\
\hline Distance by InjA & 20.84 & 0.65 & 20.35 & 76.38 & 96.66 \\
\hline Direction by InjA & left & right & right & right & right \\
\hline Distance by InjB & 96.49 & 76.13 & 56.12 & 0.59 & 20.21 \\
\hline Direction by InjB & left & left & left & left & right \\
\hline Relay based on & InjA & InjA & InjA & InjB & InjB \\
\hline Fault locaiton & zone1 & Zone2 & Zone3 & zone4 & zone5 \\
\hline Breaker open & 1 & 1,2 & 2,3 & 3 & 4 \\
\hline
\end{tabular}

Table 3 Fault location results and circuit breaker behavior when bus bar between CB3 and F3 is replaced by cable

Results in Table 3 are derived in the situation that the bus bar section between CB3 and F3 is replaced with cable. Injection B sees incorrect fault distance for F1, F2 and F3 and injection A sees incorrect fault distance for F4 and F5. However, according to the algorithm, the breaker operation decisions for F1-F3 are made based on the fault location results from Injection A and Injection B covers the F4 and F5. As in the similar situations, when other sections of bus bars are replaced with cables, correct CBs are tripped to isolate the faults. Table 4 shows the results when bus bar between CB3 and InjB is replaced and Table 5 shows the results when bus bar between CB2 and InjA is replaced.

\begin{tabular}{|c|c|c|c|c|c|}
\hline & F1 & F2 & F3 & F4 & F5 \\
\hline Distance by InjA & 20.08 & 0.64 & 20.21 & 76.05 & 95.98 \\
\hline Direction by InjA & left & right & right & right & right \\
\hline Distance by InjB & 96.82 & 76.28 & 56.69 & 0.71 & 19.95 \\
\hline Direction by InjB & left & left & left & left & right \\
\hline Relay based on & InjA & InjA & InjA & InjB & InjB \\
\hline Fault locaiton & zone1 & Zone2 & Zone3 & zone4 & zone5 \\
\hline Breaker open & 1 & 1,2 & 2,3 & 3 & 4 \\
\hline
\end{tabular}

Table 4 Fault location results and circuit breaker behavior when bus bar between CB3 and InjB is replaced by cable

\begin{tabular}{|c|c|c|c|c|c|}
\hline & F1 & F2 & F3 & F4 & F5 \\
\hline Distance by InjA & 19.67 & 0.65 & 20.33 & 40.25 & 62.07 \\
\hline Direction by InjA & left & right & right & right & right \\
\hline Distance by InjB & 97.55 & 76.02 & 19.75 & 0.59 & 20.44 \\
\hline Direction by InjB & left & left & left & left & right \\
\hline Relay based on & InjA & InjA & InjA & InjB & InjB \\
\hline Fault locaiton & zone1 & Zone2 & Zone3 & zone4 & zone5 \\
\hline Breaker open & 1 & 1,2 & 2,3 & 3 & 4 \\
\hline
\end{tabular}


Table 5 Fault location results and circuit breaker behavior when bus bar between CB2 and InjA is replaced by cable

All the tests results (from Table 2 to Table 5) show that even if in the system restoration situation, the proposed impedance based protection scheme can still work correctly without knowing the impedance of cable that is added in the marine power system.

\section{Conclusions}

A power system protection scheme based on impedance estimation using multiple triangular current injections at different position of power system is proposed. This method is tested in a $30 \mathrm{~kW}, 400 \mathrm{~V}$, twin bus DC zonal marine power distribution system demonstrator. Accompanied with Bus Interface Unit (BIU), the supply voltage of the load can be maintained constant during fault situation. The short circuit faults on the distribution bus bar can be detected very quickly using the system voltage drop information and located accurately using impedance estimation within 200ms after fault is detected. This reduced influence of system fault to minimum level.

Compared with traditional current differential protection, this method is more intelligent and able to protect the whole power system without requiring communication between current transducers. This will improve the survivability of modern "more" electric or even "all" electric ship power system. According to the test results, the algorithm works even in the system restoration situation that one section of the bus bar is replaced with a cable which has much larger and uncertain impedance value than the bus bar.

\section{References}

1 Ericsen, T., Hingorani, N., and Khersonsky, Y.: 'Power electronics and future marine electrical systems', IEEE Trans. Industry Applications, 2006, 42, (1), pp. 155-163

2 Baran, M., and Mahajan, N.: 'System reconfiguration on shipboard DC zonal electrical system'. IEEE. Int. Conf. Electric Ship Technologies Symposium, 2005, pp. 86-92

3 Christopher, E., Sumner, M., Thomas, D., Wang, X.H., and Wildt, F.: 'Fault location in a zonal DC marine power system using active impedance estimation'. Int. Conf. Energy Conversion Congress and Exposition, 2010, pp. 3050-3054

4 Thomas, D., Sumner, M., Coggins, D., Wang, X.H., Wang, J., and Geertsma, L.: 'Fault location for DC marine power system'. IEEE. Int. Conf. Electric Ship Technologies Symposium, 2005, pp. 456-460

5 Fletcher, S., Elders, I. M., Norman, P., Galloway, S., Booth, C., and Burt, G.: 'The impact of incorporating skin effect on the fault analysis and protection system performance of DC marine and aerospace power systems'. Int. Conf. Developments in Power System Protection (DPSP), 2010, pp. 1-5

6 Fletcher, S., Norman, P., Galloway, S., and Burt, G.: 'Mitigation against overvoltage on a DC marine electrical system'. IEEE. Int. Conf. Electric Ship Technologies Symposium, 2009, pp. 420-427 
7 Ciezki, J., and Ashton Robert.: ' Selection and stability issues associated with a navy shipboard DC zonal electric distribution system’, IEEE Trans. Power Delivery, 2000, 15, (2), pp. 665-669

8 Hanfner, J., and Jacobson, B.: 'Proactive hybrid HVDC breakers - a key innovation for reliable HVDC grids'. Int. Conf. Integrating Supergrids and Microgrids, 2011, pp.1-8

9 Blackburn, J. L., and Domin, T. J.: 'Protective relaying principles and applications' ( CRC Press, 2006, $3^{\text {rd }}$ edn)

10 Booth, C., Dudgeon, G., McDonald, J., Kinson, A., and Hill, J.: 'Protection of modern marine power systems: challenges and solutions'. Int. Conf. Developments in Power System Protection, 2004, pp. 825-828

11 McMurdo, J.N., and Weller, G.C.: ‘Applications of digital differential protection'. Int. Conf. Developments in Power System Protection, 1993, pp. 115-118

12 Thomas, D., Christopoulos, C., Tang, Y., Galen, P., and Stokoe, J.: 'Single ended travelling wave fault location scheme based on wavelet analysis’. Eighth IEE Int. Conf. Developments in Power System Protection, 2004, pp. 196 - 199

13 Thomas, D., Christopoulos, C., Tang, Y., and Galen, P.: 'Validation of a novel unit protection scheme based on superimposed fault currents'. Seventh IEE Int. Conf. Developments in Power System Protection, 2001, pp. 198-188

14 Thomas, D., Corvalho, R., and Pereira, E.: 'Fault location in distribution systems based on travelling waves'. Int. Conf. Power Tech, Bologna, 2003, pp. 5-9

15 Cuzner, R.M., and Venkataramanan, G.: 'The status of DC micro-grid protection'. IEEE Industry Applications Society Meeting, 2008, pp. 1-8

16 Oppenheim, A. V., and Schafer, R. W.: 'Discrete-time signal processing' (Upper Saddle River Prentice-Hall Press, 1999) pp. $467-471$

17 Miller, S. J.: 'The method of least squares'. Mathematics Department Brown University Providence, RI 02912

18 Sumner, M., Palethorpe, B., and Thomas, D.W.P.: 'Impedance measurement for improved power quality-part 1: the measurement technique' IEEE Trans. Power Delivery, 2004, 19, (3), pp. 1442-1448

19 Sumner, M., Palethorpe, B., and Thomas, D.W.P.: 'Impedance measurement for improved power quality-part 2: a new technique for stand-alone active shunt filter control' IEEE Trans. Power Delivery, 2004, 19, (3), pp. 1457-1463 